\title{
Clarificación combinada y evaluación sensorial de jugo de marañón (Anacardium occidentale L.)
}

\section{Combined clarification and sensory evaluation of cashew juice (Anacardium occidentale L.)}

\author{
Guillermo Arrázola P, ${ }^{1 *}$ Ph.D, Armando Alvis B, ${ }^{1}$ Ph.D, Jorge Osorio M, ${ }^{1}$ M.Sc. \\ ${ }^{1}$ Universidad de Córdoba, Facultad de Ingenieria, Departamento de Ingenieria de Alimentos. Grupo \\ de Investigación Procesos y Agroindustria de Vegetales, Montería, Colombia. ${ }^{*}$ Correspondencia: \\ guillermo.arrazola@ua.es
}

Recibido: Julio de 2012; Aceptado: Junio de 2013.

\section{RESUMEN}

Objetivo. Obtener jugo clarificado de marañón, evaluar sensorialmente jugos clarificados optimizados y establecer diferencias químicas entre el jugo integral y el clarificado. Materiales y métodos. Los pseudofrutos se separaron manualmente de la nuez, fueron seleccionados y lavados, luego se realizó escaldado y extracción del jugo. El jugo integral y el de mayor aceptación fueron caracterizados químicamente. Los jugos se evaluaron sensorialmente (aceptación) con una escala hedónica de 9 puntos y una prueba de ordenamiento por 30 catadores. Se empleó un diseño factorial de tres niveles combinado con la metodología de superficie de respuesta; las características químicas de los jugos se analizaron por prueba de homogeneidad de varianzas de Levene y la prueba T-Student de comparación de medias para muestras independientes. Resultados. Los jugos evaluados sensorialmente no presentaron diferencias estadísticas significativas entre sí ( $p \leq 0.05, p \leq 0.01)$; sin embargo el mayor porcentaje de aceptación fue del tratamiento $0.20 \% \mathrm{p} / \mathrm{v}$ Rapidasa ${ }^{\circledR} \mathrm{CX}$ y 14.27 horas a $30^{\circ} \mathrm{C}$, se presentaron diferencias estadísticas significativas para las variables $\mathrm{pH},{ }^{\circ} \mathrm{Brix}$, azúcares reductores y ácido ascórbico ( $\mathrm{s} \leq 0.05)$; además, el contenido de ácido ascórbico se redujo notablemente en un $41.01 \%$ con respecto al jugo inicial. Conclusiones. El jugo clarificado con alto contenido de vitamina C, obtenido por tratamiento enzimático, constituye una forma de aprovechamiento agroindustrial del pseudofruto, teniendo aceptación para su consumo y sin presentar astringencia, con buen sabor, aroma, mediante tratamiento $0.20 \% \mathrm{p} / \mathrm{v}$ Rapidasa ${ }^{\circledR} \mathrm{CX}$ y 14.27 horas a $30^{\circ} \mathrm{C}$.

Palabras clave: Composición química, evaluación, extracción de jugo (Fuente: CAB). 


\begin{abstract}
Objective. Obtain clarified cashew juice, in order to sensorially evaluate clarified and optimized juice and establish chemical differences between the integral and the clarified juice. Materials and methods. The pseudo fruits were manually separated from the nut and were selected and washed, then scalded and the juice was extracted. The natural juice and the most accepted one were chemically characterized. Juices submitted to sensory evaluation (acceptance) with a 9-point hedonic scale and a test system for 30 tasters. A three-level factorial design combined with response surface methodology was used. The chemical characteristics of juices were analyzed by Levene's test for homogeneity of variances and T-Student test for comparison of means for independent samples. Results. The evaluated juices displayed no statistically significant differences $(p \leq 0.05, p \leq 0.01)$, but the highest percentage of acceptance was for the juice treated with $0.20 \% \mathrm{w} / \mathrm{v}$ Rapidase ${ }^{\circledR} \mathrm{CX}$ and 14.27 hours at $30^{\circ} \mathrm{C}$, differences were statistically significant for $\mathrm{pH},{ }^{\circ} \mathrm{Brix}$, reducing sugars and ascorbic acid $(\mathrm{p} \leq 0.05)$, furthermore, the content of ascorbic acid was significantly reduced by $41.01 \%$ compared to the initial juice. Conclusions. The clarified juice with high vitamin $\mathrm{C}$ content, obtained by enzymatic treatment, is a way of agro-industrial use of the pseudo fruit, having been accepted for consumption, and non-astringent, with good flavor, aroma, by treatment with $0.20 \% \mathrm{w} / \mathrm{v}$ Rapidase ${ }^{\circledR} \mathrm{CX}$ and 14.27 hours at $30^{\circ} \mathrm{C}$.
\end{abstract}

Key words: Chemical composition, evaluation, juice extraction, (Source: $C A B$ ).

\title{
INTRODUCCIÓN
}

El marañón (Anacardium occidentale $\mathrm{L}$ ) es una fruta que se encuentra bien establecida en todo el mundo tropical, encontrándose desde los Estados Unidos hasta África del sur. Entre los países de mayor concentración del cultivo además de Brasil, se tiene a la India, Kenia, Mozambique y Tanzania $(1,2)$. En Colombia, el objetivo central del cultivo de marañón es la nuez, con una producción aproximada de 5000 toneladas (3).

Mientras que la nuez del marañón es de un alto valor en la industria de alimentos, la manzana (pseudofruto) se considera a menudo como un subproducto con una producción estimada en el año 2006 de 30 millones de toneladas métricas, más allá de la piña y del mango (4). Parte de la producción de pseudofruto se desperdicia, pudriéndose al lado de los arboles después de la cosecha de nuez, sin embargo, otra parte se transforma en jugo, dulces etc. (5). A pesar del potencial de pseudofruto como materia prima para diversos productos, alrededor del $90 \%$ de su producción se desecha cada año, debido a que son muy perecederos y el negocio principal es la comercialización de las almendras (6). Sin embargo, es rica en vitamina C y compuestos fenólicos, sustancias con alto potencial antioxidante, que han atraído el interés de diferentes grupos de investigación (7).

El bajo consumo en el mercado internacional de jugo de marañón es atribuible al uso de preservantes químicos, a la falta de mejora de procesos tecnológicos, a la astringencia por los taninos presentes (8) y presencia de sólidos en suspensión (9). Durante la obtención de jugo del pseudofruto de marañón con la finalidad de remover los fenoles, taninos y eliminar la pectina se ha empleando enzimas como pectinasas y tanasa asociadas a la microfiltración (8). Además, se han evaluado agentes clarificantes como almidón comercial y gelatina $(9,10)$.

Por otra parte, se debe tener en cuenta que durante el procesamiento industrial, ocurren modificaciones en los componentes de los frutos que afectan sus propiedades sensoriales, tales como textura, sabor, aroma y valor nutritivo; por lo tanto, un proceso adecuado de las frutas ocasiona pequeñas pérdidas de estas cualidades. Por lo tanto es necesario que las técnicas de procesamiento y conservación de los jugos sean eficaces de mantener en los productos procesados, las características originales de las frutas (11).

En esta investigación se planteó como objetivo la obtención de jugo clarificado a partir del pseudofruto del marañón empleando agente floculante y enzima comercial, evaluando sensorialmente el jugo obtenido y estableciendo diferencias fisicoquímicas entre el jugo integral y el clarificado; contribuyendo así a crear nuevas alternativas para el uso de la pseudofruto y por lo tanto, para reducir las pérdidas.

\section{MATERIALES Y MÉTODOS}

Material vegetal. La investigación se realizó en el Laboratorio de Nutrición y el Laboratorio de Microbiología y Biotecnología de Alimentos de la Universidad de Córdoba, sede Berástegui, municipio de Ciénaga de Oro, localizada geográficamente en las coordenadas 8040'26" 
latitud Norte y $75^{\circ} 46^{\prime} 44^{\prime \prime}$ longitud Oeste con respecto al meridiano de Greenwich.

Los pseudofrutos de marañón de los cultivares Martinica, Antillana y Amazónico, se cosecharon en el corregimiento de Pisa Bonito (Chinú, Córdoba), libres de daños mecánicos y en estado de madurez adecuado. La nuez se separó del pseudofruto (manzana) manualmente. La recolección de los pseudofrutos se realizó en las horas de la mañana y fueron transportados hasta el laboratorio, donde se seleccionaron y lavaron.

Obtención del jugo. El jugo integral se consiguió mediante prensado empleando un extractor manual. Después se tamizó a través de un filtro prensa Corel 234 Modelo 2009 , equipado con malla de polipropileno con multifilamento, utilizando además una precapa con tierra diatomeas (Hy flow y carbón activo en polvo tipo Darko 50). Para el proceso de clarificación de jugo, los pseudofrutos fueron inicialmente escaldados en agua a $100^{\circ} \mathrm{C}$ por 3 min; enfriadas a temperatura de laboratorio y luego prensadas (12).

Caracterización química del jugo de marañón. Para determinar las características fisicoquímicas del jugo integral y clarificado de marañón se realizaron los siguientes análisis: $\mathrm{pH}$, según el método 981.12 (adaptado), usando un potenciómetro Oakton Ion 510 Series; Acidez titulable, según el método 942.15 (adaptado), empleando una solución de $\mathrm{NaOH} 0.1 \mathrm{~N}$, el resultado se expresó como porcentaje de ácido cítrico; Sólidos solubles ( ${ }^{\circ}$ Brix), según el método 932.12 (adaptado) (13), utilizando el refractómetro Bertucci modelo A36P. Determinación de azúcares reductores, método DNS (Ácido 3,5-dinitrosalicílico) de la Universidad Nacional de Colombia (adaptado); taninos, método 952.03 (Folin - Denis) (adaptado) (13) y ácido ascórbico, método fotométrico con 2,4-dinitrofenilhidrazina (14). Los resultados fueron expresados como $\mathrm{mg}$ de azúcares reductores, de ácido ascórbico y de ácido tánico en $100 \mathrm{~mL}$ de jugo, respectivamente. Se empleó un espectrofotómetro GENESIS 20 UV-Vis, realizando nueve repeticiones por análisis y tres replicas analíticas a cada muestra.

Clarificación del jugo de marañón. Para controlar la astringencia del jugo primero se adicionó solución de gelatina ${ }^{\circledR}$ al $1 \%$ p/v, se agitó y dejó en reposo por 10 minutos a temperatura de laboratorio $\left(30 \pm 1^{\circ} \mathrm{C}\right)$, se agitó nuevamente, dejándose en reposo por 30 minutos y se filtró a través de una tela tipo malla $(9,15)$. Luego, al filtrado se le adicionó la enzima comercial
Rapidasa $^{\circledR}$ CX $(0,1 ; 0,15$ y $0,2 \% \mathrm{p} / \mathrm{v})$, a tres tiempos de acción $(6,12$ y $18 \mathrm{~h})$, a diferentes temperaturas de acción $\left(20 ; 25\right.$ y $\left.30^{\circ} \mathrm{C}\right)$ y un $\mathrm{pH}$ ajustado de 4.02 .

Una vez concluido el tiempo de acción enzimática, los jugos clarificados se filtraron a vacío usando papel filtro Whatman No 1 (16). El jugo filtrado se embotelló en recipientes plásticos (200 $\mathrm{mL})$, fueron pasteurizados $\left(72^{\circ} \mathrm{C} / 30 \mathrm{~s}\right)$ y luego refrigerados $\left(4^{\circ} \mathrm{C}\right)$, tomando las muestras para los respectivos análisis.

Evaluación de la actividad enzimática. Mediante espectrofotometría se determinó la concentración de azucares reductores (método DNS) y grado de clarificación $(16,17)$ de los jugos tratados enzimáticamente. A los resultados se les realizó análisis de regresión y optimización.

Análisis sensorial. Se seleccionaron tres tratamientos del proceso de optimización y se evaluaron una escala hedónica (18), aplicando una prueba descriptiva con una escala estructurada de nueve (9) puntos (donde $1=$ disgusta muchísimo, 5 = indiferente y $9=$ gusta muchísimo) y una prueba de ordenamiento, los resultados fueron analizados por Anova y presentados en forma gráfica para obtener una idea cualitativa y cuantitativa del espectro o configuración del nivel de agrado de los jugos clarificados.

Diseño Experimental. Se utilizó un diseño factorial a tres niveles para el ajuste de un modelo cuadrático, combinado con la metodología de superficie de respuesta. La variables independientes fueron concentración enzimática $\left(x_{1}\right)$, temperatura de acción $\left(x_{2}\right)$ y tiempo de acción $\left(\mathrm{x}_{3}\right)$, cada una con tres niveles. Un total de 32 diferentes combinaciones (incluyendo seis replicas del punto central) fueron seleccionados en orden aleatorio. Las variables dependientes (y) fueron concentración de azúcares reductores y grado de clarificación (absorbancia). El software estadístico comercial Desing Expert Versión 6.0.1 (Stat-Ease, Minneapolis, USA) fue empleado para generar el diseño experimental, los análisis estadísticos y modelo de regresión.

Las características fisicoquímicas de los jugos integral y clarificado de mayor aceptación se analizaron por prueba de homogeneidad de varianzas de Levene y la prueba T-Student de comparación de medias para muestras independientes. 


\section{RESULTADOS}

Fisicoquímicas del jugo de marañón. En la tabla 1 , se muestran los resultados promedios de los análisis fisicoquímicos obtenidos en el jugo integral de marañón.

Tabla 1. Análisis fisicoquímicos del jugo integral y clarificado de marañón.

\begin{tabular}{lcc}
\hline \multicolumn{1}{c}{ Análisis Fisicoquímicos } & $\begin{array}{c}\text { Jugo Integral } \\
\text { de marañón }\end{array}$ & $\begin{array}{c}\text { Jugo } \\
\text { Clarificado de } \\
\text { marañón }\end{array}$ \\
\hline $\mathrm{pH}$ & $4.03 \pm 0.04^{\mathrm{a}}$ & $4.15 \pm 0.00^{\mathrm{b}}$ \\
\hline Acidez & $0.32 \pm 0.03^{\mathrm{a}}$ & $0.39 \pm 0.01^{\mathrm{a}}$ \\
\hline${ }^{\circ}$ Brix (Sólidos solubles) & $14.11 \pm 0.78^{\mathrm{a}}$ & $8.33 \pm 0.58^{\mathrm{b}}$ \\
\hline Azucares reductores $(\mathrm{mg} / 100 \mathrm{~mL})$ & $60.31 \pm 7.80^{\mathrm{a}}$ & $242.67 \pm 4.62^{\mathrm{b}}$ \\
\hline Ácido tánico $(\mathrm{mg} / 100 \mathrm{~mL})$ & $0.71 \pm 0.56^{\mathrm{a}}$ & $0.27 \pm 0.01^{\mathrm{a}}$ \\
& & \\
\hline Ácido ascórbico $(\mathrm{mg} / 100 \mathrm{~mL})$ & $210.08 \pm 20.04^{\mathrm{a}}$ & $123.93 \pm 1.42^{\mathrm{b}}$
\end{tabular}

a, b Promedios con la misma letra son estadísticamente iguales para la prueba de homogeneidad de varianzas de Levene y la prueba T-Student de comparación de medias para muestras independientes.

Obtención de jugo clarificado de marañón. Los pseudofrutos que fueron seleccionados para el proceso de obtención de jugo clarificado, se sometieron a escaldado como método para la estabilización de la materia prima. Se extrajo el jugo por prensado mecánico obteniendo un rendimiento del $60 \%$ y generándose un bagazo. El jugo fresco obtenido de los pseudofrutos escaldados fue envasado en recipientes plásticos y mantenidos congelación $\left(-18^{\circ} \mathrm{C}\right)$ para los posteriores análisis.

Más adelante, el jugo integral fue descongelado hasta llevarlo a temperatura de laboratorio, donde se les realizó el tratamiento con la solución de gelatina, observándose la formación de flóculos que se retiraron por filtración. Luego los jugos fueron expuestos a los diferentes tratamientos enzimáticos. Seguidamente, se evaluó la actividad enzimática mediante la obtención de los jugos clarificados y pasteurizados para la determinación de azucares reductores y el grado de clarificación de los jugos.

Evaluación de la actividad enzimática. En la tabla 2, se muestran los coeficientes de las ecuaciones de regresión ajustadas para el contenido de azucares reductores y grado de clarificación, utilizados para establecer la influencia de la concentración de la enzima, la temperatura y tiempo de acción sobre la obtención de jugo clarificado de marañón. Como puede observarse las variables tiempo y temperatura de acción son altamente significativos para el contenido de azúcares reductores y grado de clarificación $(p \leq 0.01)$. La concentración enzimática no fue significativa para
Tabla 2. Información estadística para las variables de respuesta azúcares reductores y grado de clarificación.

\begin{tabular}{ccc}
\hline Factor & $\begin{array}{c}\text { Azúcares reductores } \\
(\mathbf{m g} / \mathbf{1 0 0} \mathbf{~} \mathbf{~ j u g o})\end{array}$ & $\begin{array}{c}\text { Grado de } \\
\text { clarificación } \\
\text { (Abs.) }\end{array}$ \\
\hline costante & 220.76 & 0.030 \\
$\mathrm{E}$ & $3.36 \mathrm{NS}$ & $-1.056 \times 10^{-3 * * *}$ \\
$\mathrm{~T}$ & $13.81 * * *$ & $-1.611 \times 10^{-3 * * *}$ \\
$\mathrm{t}$ & $24.90 * * *$ & $-2.944 \times 10^{-3 * * *}$ \\
$\mathrm{E}^{2}$ & $-1.40 \mathrm{NS}$ & $7.258 \times 10^{-4} \mathrm{NS}$ \\
$\mathrm{T}^{2}$ & $-2.46 \mathrm{NS}$ & $3.925 \times 10^{-4} \mathrm{NS}$ \\
$\mathrm{C}^{2}$ & $-24.28 * * *$ & $2.392 \times 10^{-3 * * *}$ \\
Tt & $-1.57 * *$ & $1.667 \times 10^{-4 * *}$ \\
ET & $-5.09 \mathrm{NS}$ & $1.000 \times 10^{-3} \mathrm{NS}$ \\
Et & $-1.06 \mathrm{NS}$ & $-3.333 \times 10^{-4} \mathrm{NS}$ \\
$\mathrm{R}^{2}$ & 0.9364 & 0.8677 \\
\hline
\end{tabular}

E: concentración enzima $(\% \mathrm{p} / \mathrm{v}) ; \mathrm{T}$ : temperatura de acción $\left({ }^{\circ} \mathrm{C}\right) ; \mathrm{t}$ : tiempo de acción (h). ${ }^{*} * \mathrm{a}=5 \%$; $* * * \mathrm{a}=1 \%$; NS: No significativo, $\mathrm{R}^{2}=$ coeficiente de determinación.

el contenido de azúcares reductores, mientras que para el grado de clarificación esta variable fue significativa $(p \leq 0.01)$. Por otra parte, el coeficiente cuadrático del tiempo de acción fue altamente significativo $(p \leq 0.01)$, mientras que el coeficiente cuadrático de la temperatura de acción y la concentración de la enzima no fueron significativas para azucares reductores y grado de clarificación $(p>0.01)$.

Los análisis estadísticos mostraron que la interacción temperatura y tiempo de acción fue significativa para el contenido de azucares reductores y grado de clarificación $(p \leq 0.05)$, mientras que las demás interacciones no fueron significativas $(p>0.05)$.

Las ecuaciones de regresión ajustadas se utilizaron para obtener los gráficos de las superficies de respuesta y visualizar los efectos de la concentración enzimática, temperatura y tiempo de acción sobre las variables analizadas.

Azúcares reductores $=-155.570+25.365 t+$ $10.351 T-0.674 t^{2}-0.169 t T$

En la figura 1a, se aprecia que la concentración de azúcares reductores incrementa con el aumento del tiempo y la temperatura de la acción. Asimismo, en la figura 1b, se observa aumento de los azúcares reductores con el incremento de la concentración enzimática; sin embargo estadisticamente esto no fue significativo $(p>0.01)$. La máxima cantidad de azúcares reductores en el jugo clarificado se obtiene al combinar $0.15 \%$ de Rapidasa ${ }^{\circledR}$ CX, temperatura 


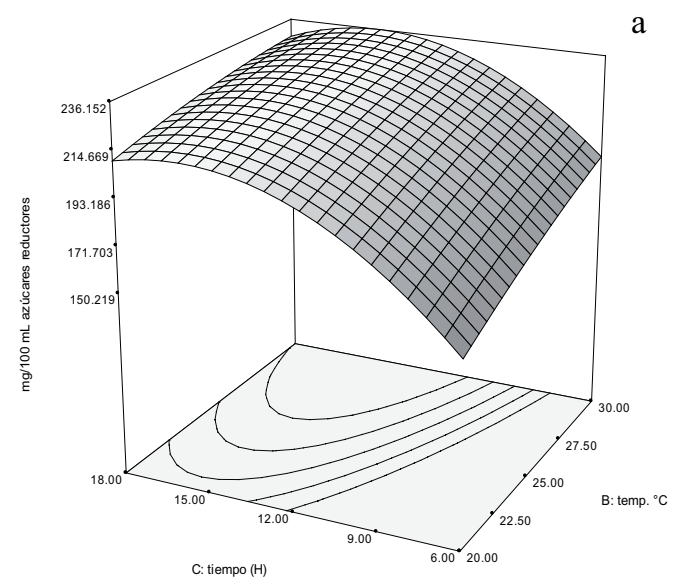

a

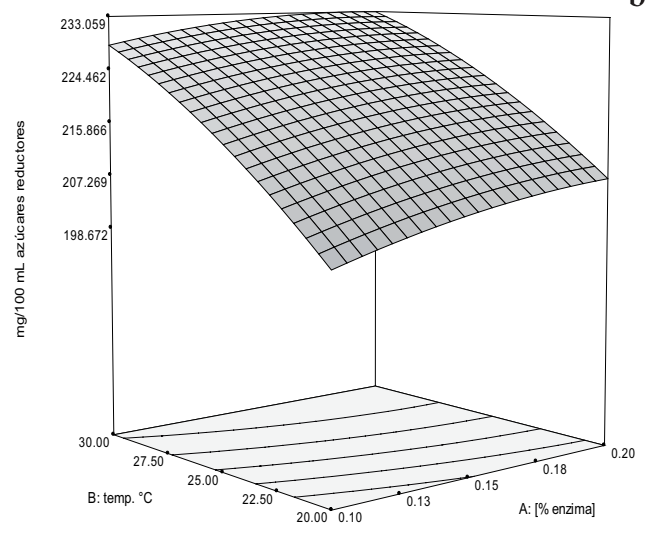

Figura 1. Superficie de respuesta para el contenido de azúcares reductores de jugo clarificado como una función (a) temperatura y tiempo (concentracion enzimatica $0.15 \% \mathrm{p} / \mathrm{v}$ ); (b) concentración enzima y temperatura (12 horas)

de acción de $30^{\circ} \mathrm{C}$ y un tiempo de 12 horas, con un promedio de $249.091 \mathrm{mg} / 100 \mathrm{~mL}$. La acción de la enzima Rapidasa ${ }^{\circledR}$ CX fue demostrada en cada uno de los tratamientos al compararse con el contenido de azúcares del jugo patrón $(63.177 \mathrm{mg} / 100 \mathrm{~mL})$.

La ecuación del modelo ajustado para el grado de clarificación de los jugos obtenidos fue la siguiente:

Grado de clarificación $=0.078-3.002 \times 10^{-}$ ${ }^{3} t-0.081 E-1.307 \times 10^{-3} T+6.645 \times 10^{-5} t^{2}+$ $3.333 \times 10^{-5} t T$

El jugo clarificado es un jugo natural que tiene menos solidos no solubles en suspención, por lo cual no presenta turbidez. En la figura 2, se representa la variación del grado de clarificación de los jugos de marañón en función del tiempo y la temperatura de acción a las diferentes concentraciones de la enzima pectinasa. El mayor grado de clarificación se observó al emplear $0.15 \% \mathrm{p} / \mathrm{v}$ de enzima, temperatura de $25^{\circ} \mathrm{C}$ y un tiempo de 12 horas. En la figura también se evidencia que los valores de absorbancia decrecen con el incremento del tiempo. El grado de clarificación presentó la más baja absorbancia al tiempo de acción más alto. También, se aprecia que los valores de absorbancia disminuyeron con el aumento de la temperatura de acción a una concentración enzimatica fija. La acción de la enzima Rapidasa ${ }^{\circledR}$ CX fue constatada en cada uno de los tratamientos teniendo como referencia la absorbancia del jugo patrón (0.128).

La absorbancia al medir el grado de clarificación de los jugos es inversamente proporcional a la concentración de la enzima. Asimismo, la absorbancia de los jugos clarificados disminuye con el aumento del tiempo y temperatura de acción, con respecto al aumento de la concentración enzimática.
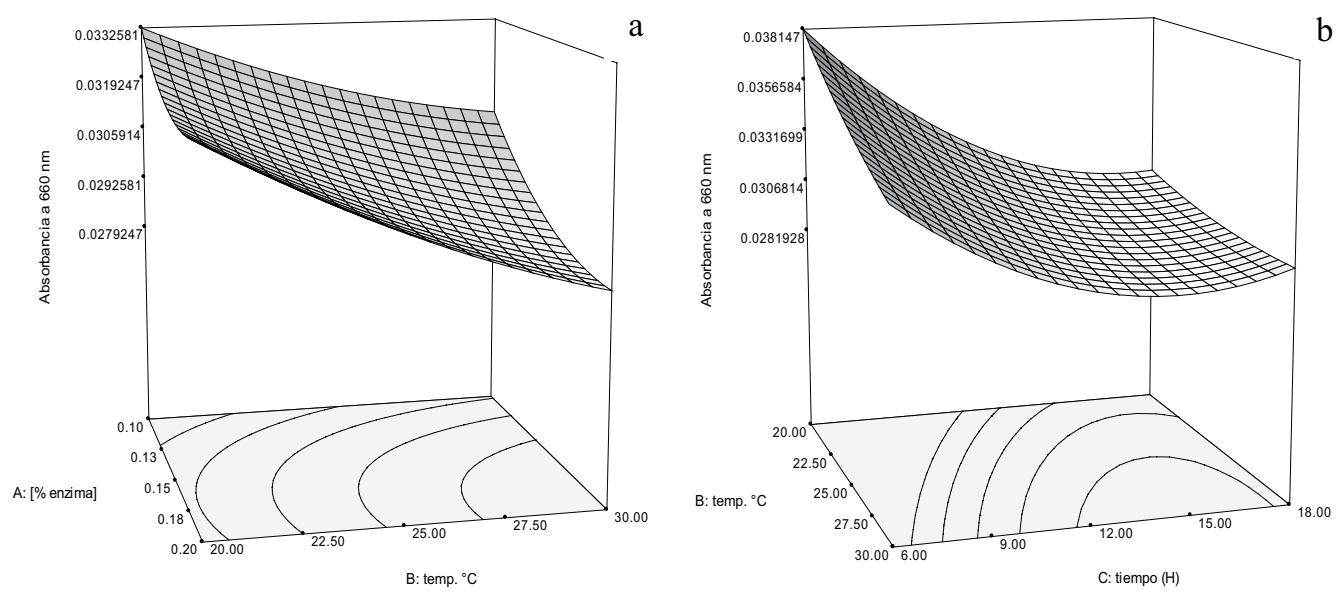

Figura 2. Superficie de respuesta para el grado de clarificación de jugo clarificado como una función (a) concentración enzima y temperatura (12 horas); (b) temperatura y tiempo (concentracion enzimatica $0.15 \% \mathrm{p} / \mathrm{v})$. 
Optimización del proceso de Clarificación. Se determinaron las condiciones óptimas para los resultados deseados como contenido de azúcares reductores (máximo) y grado de clarificación (mínima absorbancia). Dado que la respuesta óptima para cada variable dependiente no caen exactamente en la misma región, se realizó la intercepción de las superficies de respuestas.

En la tabla 3, se registran las soluciones numéricas del proceso de optimización arrojado por el software estadístico teniendo en cuenta el criterio de selección antes mencionado. En ella se observa cinco soluciones con la respuesta calculada para azúcares reductores entre 236.646 a $236.568 \mathrm{mg} / 100 \mathrm{~mL}$ y absorbancia de 0.027 ; con un porcentaje de conveniencia del 91.9 al $92 \%$. A partir de estos resultados se escogieron tres tratamientos optimizados teniendo en cuenta la concentración enzimática y el tiempo de acción. A las soluciones con igual concentración enzimática se les determinó un tiempo medio de acción. Los tratamientos para evaluar el nivel de agrado de los jugos clarificados correspondieron a: $0.18 \%$ p/v Rapidasa ${ }^{\circledR}$ CX por 14.37 horas (referencia: J_030); $0.19 \% \mathrm{p} / \mathrm{v}$ Rapidasa ${ }^{\circledR} \mathrm{CX}$ por 14.25 horas (referencia: J_205) y $0.20 \%$ p/v Rapidasa ${ }^{\circledR}$ y 14.27 horas (referencia: J_711).

Tabla 3. Soluciones numéricas del proceso de optimización para la obtención de jugo clarificado de marañón.

\begin{tabular}{cccccc}
\hline No Tiempo & $\begin{array}{c}\text { Concentración } \\
\text { enzimática } \\
\text { (\%)/v) }\end{array}$ & $\begin{array}{c}\text { Azúcares } \\
\text { Reductores } \\
\text { (mg/ } \\
\mathbf{1 0 0} \mathbf{~ m L})\end{array}$ & $\begin{array}{c}\text { Grado de } \\
\text { clarificación } \\
\text { (Abs 660 } \\
\mathbf{n m} \text { ) }\end{array}$ & Conveniencia \\
\hline 1 & 14.27 & 0.19 & 236.568 & 0.027 & 0.920 \\
2 & 14.24 & 0.19 & 236.558 & 0.027 & 0.920 \\
3 & 14.30 & 0.20 & 236.498 & 0.027 & 0.920 \\
4 & 14.25 & 0.20 & 236.479 & 0.027 & 0.920 \\
5 & 14.37 & 0.18 & 236.646 & 0.027 & 0.919 \\
\hline
\end{tabular}

Evaluación sensorial de los jugos clarificados de marañón. El análisis sensorial en la escala estructurada mostró que el $63.33 \%$ de los catadores al menos referenció gustarle ligeramente el jugo rotulado como J_711, el $60.00 \%$ el jugo J_030 y $56.67 \%$ el jugo J_025. En la prueba de ordenamiento el $60.00 \%$ de los panelistas expresaron sentir mayor agrado con el jugo J_711, seguido del jugo J_025 y por último el J_030 con el 26.67 y $13.33 \%$, respectivamente.

El análisis de varianza realizado a los resultados de la evaluación sensorial mediante la escala estructurada ( $F$ calculado $=1.330$ ) y la prueba de ordenamiento $(F$ calculado $=1.243$ ) de los tres jugos escogidos del proceso optimización no mostraron diferencias estadísticas significativas, para una significancia del $5 \%$. Teniendo en cuenta los resultados obtenidos en la prueba de aceptación se escogió como mejor tratamiento al jugo J_711 que se obtuvo por el tratamiento de $0.20 \% \mathrm{p} / \mathrm{v}$ Rapidasa ${ }^{\circledR}, 14.27 \mathrm{~h}$ a $30^{\circ} \mathrm{C}$.

Características fisicoquímicas del jugo clarificado de marañón. La prueba de homogeneidad de varianzas de Levene y la prueba T-Student de comparación de medias para muestras independientes entre el jugo integral y clarificado de marañón que presentó mayor aceptación en la evaluación sensorial, mostraron que solo las variables $\mathrm{pH},{ }^{\circ} \mathrm{Brix}$, azúcares reductores y ácido ascórbico presentan diferencias estadísticas significativas (Tabla 1).

El pH del jugo clarificado de marañón aumentó con respecto al jugo integral. También se observa que la acidez titulable se incrementó, lo que está relacionado con el aumento de azucares reductores, sin embargo esta variación no es significativa estadísticamente $(p \leq 0.05)$. El contenido de azucares reductores incrementó significativamente debido a la acción enzimática sobre la pectina presente en el jugo integral, esta actividad conduce a la liberación de moléculas de ácido galacturónico.

Por otra parte, se aprecia una disminución en los sólidos solubles en el jugo clarificado con respecto al jugo integral. En el mismo sentido, el contenido de ácido tánico disminuyó de $0.707 \mathrm{mg} / 100 \mathrm{~mL}$ en el jugo integral a $0.268 \mathrm{mg} / 100 \mathrm{~mL}$ en el jugo clarificado; con lo cual se reduce la astringencia del jugo, aumenta su grado de clarificación y nivel de agrado. El contenido de ácido ascórbico se redujo notablemente en un $41.01 \%$ respecto al jugo inicial (Tabla 1).

\section{DISCUSIÓN}

Fisicoquímicas del jugo de marañón. $\mathrm{El} \mathrm{pH}$ del jugo integral de marañón presentó valores dentro del rango de $\mathrm{pH}$ reportados por otros autores para pulpa pseudofruto de marañón comercial $(3.77-4.23)(8,11,19)$ y en jugo integral de pseudofruto de marañón clon CCP-76 $(3.16-4.59)(9,20,21)$.

Los valores de acidez son menores a datos reportados entre $1.17-1.42 \%$ (20) y están dentro del rango reportado de $0.19-0.37 \%$ (21). Los ${ }^{\circ}$ Brix presentaron resultados mayores a los reportados de 7.4-12.5 ${ }^{\circ} \mathrm{Brix}(8,9,11)$ y menores a los publicados entre $16.46-17$ OBrix (20).

El contenido de azúcares reductores (60.312 $\mathrm{mg} / 100 \mathrm{~mL}$ equivalente a $6.03 \%$ ) fue menor a las concentraciones reportadas para pulpa de marañón comercial con $9.85 \%$ azúcares 
reductores (19) y mayores a los datos obtenidos para pseudofruto de marañón con porcentajes entre 1.45-1.46\% de azúcares reductores (21).

El contenido de ácido ascórbico del jugo es mayor a los determinados en pulpa de manzana de marañón comercial con valores de 135 - 203.5 $\mathrm{mg} / 100 \mathrm{~mL}(5,8,11,22)$; se encuentran dentro del rango de $206.20-252.40 \mathrm{mg} / 100 \mathrm{~mL}$ (21) y son menores a $335 \mathrm{mg} / 100 \mathrm{~mL}$ (9), reportados en jugo de marañón clon CCP-76.

El contenido de ácido tánico fue menor a los reportados para pulpa de marañón comercial (0.71-4.35 mg/100 mL) $(8,11,21)$ y jugo de marañón clon CCP-76 (9).

Obtención de jugo clarificado de marañón. Durante la extracción de jugo integral a partir de los pseudofrutos escaldados se generó un bagazo que representa aproximadamente el $40 \%$ del peso de la manzana de marañón. Para el aprovechamiento de este subproducto se ha empleado como sustrato para alimentos de animales (23) o para la obtención de productos de valor agregado (24).

La gelatina en solución acuosa, que se empleó para el control de la astringencia del jugo, se comporta como un coloide hidrófilo con carga eléctrica negativa, transformándose en otro coloide también hidrófilo con carga negativa cuando se le añade a jugo, con un valor de $\mathrm{pH}$ inferior a 4.7 donde se encuentra su punto isoeléctrico. La interacción gelatina y taninos, con ayuda de la presencia de cationes del jugo (calcio, potasio, hierro, etc.), producen la descarga del coloide y su consiguiente floculación (25). De este modo se favoreció la reducción de la astringencia de los jugos. La gelatina sin sabor presenta una mejor clarificación porque no muestra cambios significativos en las propiedades sensoriales del jugo (9).

Uno de los principales problemas encontrados en la obtención de jugo de marañón es la turbidez dedido principalmente a la presencia de pectinas. La turbidez en estos casos es dificil de eliminar excepto por despectinación enzimática. La pectinasa hidrolisa la pectina y provoca que complejos pectina-proteina floculen (17). El jugo resultante del tratamiento con la pectinasa puede tener una baja cantidad de pectina y una menor viscosidad, lo cual es ventajoso para facilitar los subsecuentes procesos (26). La hidrolisis de sustancias pecticas (pectina, protopectina, ácidos pécticos, acidos pectinícos) esta influenciada por varios factores del tratamiento enzimatico tales como tiempo, temperatura de acción y concentración de la enzima (27).
La influencia de los factores concentración de la enzima, tiempo y temperatura de acción en esta investigacion, fueron similares a los reportados para la optimización de la clarificacion de jugo de carambola (Averrhoa carambola L) (16) y para la clarificacion de jugo de nispero (Achras sapota) (17).

Características fisicoquímicas del jugo clarificado de marañón. El pH del jugo clarificado de marañón aumentó con respecto al jugo integral; así mismo se ha reportado un incremento en el $\mathrm{pH}$ del jugo clarificado de marañón por microfiltración en relación al jugo integral (8).

La disminución en el contenido de los sólidos solubles en el jugo clarificado con respecto al jugo integral es debido a degradación de pectina y precipitación de protopectina por acción enzimática; reduciendo así las sustancias que se encuentran en suspensión en el jugo clarificado final $(26,27)$.

El contenido de ácido tánico disminuyó en el jugo clarificado; la explicación para este comportamiento puede ser debido a la atracción que ejerce el agente coagulante sobre los compuestos fenólicos, propiciando la formación de coágulos y por consiguiente su precipitación (9).

La perdida de vitamina $C$ se presenta por una reacción de oxidación la cual ocurre en el jugo de fruta durante el almacenamiento, y es altamente dependiente de la cantidad de oxígeno en el espacio de cabeza o disuelto en el jugo y la temperatura de procesamiento (28). El porcentaje de pérdida en el contenido de ácido ascórbico en esta investigación es mayor al reportado en la obtención de jugo clarificado por microfiltración, donde se obtuvo una reducción del $22.96 \%$ (8) y del $14.28 \%$ (11) en relación a la fruta fresca. La reducción en el contenido de vitamina $C$ en esta investigación se atribuye al método de conservación, por someter el jugo a un tratamiento térmico como la pasterización. La pasteurización tiene como objetivo producir un alimento seguro, de alta calidad, a bajo costo, prolongar la vida de anaquel del alimento, mantener las características deseables (26) como el pH del producto y sus propiedades organolépticas. Durante el proceso de pasterización realizado, se logra conseguir la estabilidad (desoxigenación) y calidad del producto inactivando microorganismos termolábiles y enzimas deteriorantes (26, 27); sin embargo, puede afectar el contenido nutricional de los alimentos como las vitaminas. 
En conclusión las variables independientes temperatura y tiempo de acción afectaron notablemente el contenido de azúcares reductores, mientras que la variable concentración enzimática, junto con las anteriores, ejercieron influencia significativa sobre la variable respuesta grado de clarificación de los jugos clarificados. El contenido de vitamina $C$ del jugo de marañón se vio reducido por oxidación durante el procesamiento para la obtención de un jugo clarificado. Sin embargo, puede considerarse como una fuente de ácido ascórbico. Teniendo en cuenta los resultados obtenidos en la prueba de aceptación se escogió como mejor tratamiento al jugo J_711 que se obtuvo por el tratamiento de $0.20 \% \mathrm{p} / \mathrm{v}$ Rapidasa $^{\circledR}, 14.27$ horas a $30^{\circ} \mathrm{C}$.

\section{Agradecimientos}

A la Asociación de Productores de Marañón de la Sabana (Chinú-Colombia) y a la Comisión Departamental de Ciencia y tecnología CODECYT (Gobernación de Córdoba, Colombia).

\section{REFERENCIAS}

1. Galdámez A. Guía Técnica del Cultivo del Marañón. Programa Nacional de Frutas de El Salvador. Ministerio de Agricultura Y Ganadería. Gallardo (México): Instituto Interamericano de Cooperación para la Agricultura IICA; 2004.

2. Sousa de Brito E, Pessanha de Araújo M, Lin L, Harnly J. Determination of the flavonoid components of cashew apple (Anacardium occidentale) by LC-DAD-ESI/MS. Analytical, Nutritional and Clinical Methods. Food Chem 2007; 105:1112-1118.

3. Rodríguez L. Marañón un Recurso Valioso para el Aprovechamiento Industrial en Colombia. Aliméntica 2007; 5:2-5.

4. Statistical databases, FAO (FAOSTAT). [Internet]. Roma, Italy: FAO. 2007 [Actualizado 8 de enero del 2007; citado 18 de septiembre de 2010].Disponible: http://faostat.fao.org/site/567/default. aspx\#ancor.

5. Akinwale T. Cashew apple juice: its use in fortifying the nutritional quality of some tropical fruits. Eur Food Res Technol 2000; 211(3):205-207.

6. Paiva FA, Garruti DS, Silva Neto RM. Aproveitamento industrial do caju. Fortaleza: Embrapa Agroindústria Tropical/ SEBRAE; 2000.

7. Jiao B, Cassano A, Drioli E. Recent advances on membrane processes for the concentration of fruit juices: a review. J Food Eng 2004; 63:303-324.
8. Pereira D, Sabino A, Blumenberg D, Martins V, Corrêa L, Couri S. Cashew apple juice stabilization by microfiltration. Desalination 2002; 148:61-65.

9. Sindoni M, Caldera E, Pérez A, Marcano L, Parra R, Marín C. Evaluation of coagulating agents for the formulation of juice from pseudofruits of merey. Agronomía Tropical 2007; 57(1):61-65.

10. Jayalekshmy V, John P. 'Sago' - a natural product for cashew apple juice clarification. Short communication. J Trop Agric 2004; 42(1-2):67-68.

11. Cianci F, Silva L, Cabral K, Matta V. Clarification and concentration of cashew apple juice by membrane processes. Cienc Tecnol Aliment 2005; 25(3):579-583.

12. Arrázola G, Villalba M. Frutas, hortalizas y tuberculos. Perspectivas de agro industrialización. Montería, Colombia: Universidad de Córdoba; 2009.

13. Association of Official Analytical Chemists (A.O.A.C.). Official methods of analysis of the Association of Official Analytical Chemists. Washington, DC: The Association; 1990.

14. Linhares L, Donizete C, Patto C, Duarte A. Chemical, physical and enzymatic transformations of guavas treated at postharvest with calcium chlorite and 1-methylciclopropone and stored under refrigeration. Ciênc Agrotec Lavras 2007; 31(3):829-841. 
15. Ashurst PR. Producción y Envasado de Zumos y Bebidas de Frutas sin gas. 2a edición. Zaragosa, España: Acribia, S.A.; 1995.

16. Liew A, Sulaiman N, Aroua M, Megat M. Response surface optimization of conditions for clarification of carambola fruit juice using a commercial enzyme. J Food Eng 2007; $81: 65-71$.

17. Sin $H$, Yusof $S$, Sheikh $H$, Rahman R. Optimization of clarification of sapodilla juice using Response surface methodology. J Food Eng 2006; 73:313-319.

18. Anzaldúa, A. Evaluación Sensorial de los Alimentos en la Teoría y en la Práctica. Zaragoza, España: Acribia, S.A.; 1998.

19. Azoubel P, Cipriani D, El-Aouar A, Antonio $\mathrm{G}$, Xidieh $\mathrm{E}$. Effect of concentration on the physical properties of cashew juice. J Food Eng 2005; 66:413-417.

20. Guerrero R, Lugo L, Marín M, Beltrán O, León de Pinto G, Rincón F. Physicalchemical characterization of the fruit and pseudofruit of Anacardium occidentale L. (cashew) under unirrigated land conditions. Rev Fac Agron (LUZ) 2008; 25:81-94.

21. Lowor S, Agyente-Badu C. Mineral and Proximate Composition of Cashew Apple (Anacardium occidentale L.) Juice from Northern Savannah, Forest and Coastal Savannah Regions in Ghana. Am J Food Technol 2009; 4(4):154-161.
22. Janick J, Paull R. The encyclopedia of fruits and nuts. Cambridge, UK: Cambridge University Press; 2006.

23. Santos R, Santiago A, Gadelha C, Cajazeiras J, Cavada B, Martins J, Oliveira T, Bezerra G, Santos R, Freire V. Production and characterization of the cashew (Anacardium occidentale L.) peduncle bagasse ashes. J Food Eng 2007; 79(20): 1432-1437.

24. Lopes T, Rabelo M, Barros L, Saavedra $G$, Rodrigues S. Fermentation of cashew Apple juice to produce high added value products. World J Microbiol Biotechnol 2007; 23:1409-1415.

25. Molina R. Teoría de la Clarificación de mostos y vinos y sus aplicaciones prácticas. Madrid: AMV Ediciones, Mundi Prensa; 2000.

26. Rai P, Majumdar GC, DasGupta S, De S. Optimizing pectinase usage in pretreatment of mosambi juice for clarification by response surface methodology. J Food Eng $2004 ; 64: 397-403$.

27. Lee WC, Yusof S, Hamid NSA, Baharin BS. Optimizing conditions for enzymatic clarification of banana juice using response surface methodology (RSM). J Food Eng 2006, 73:55-63.

28. Oliveira M, Arraes G, Wilane R, Moreira de Souza M, Montenegro I. Storage stability of cashew Apple juice preserved by hot fill and aseptic processes. Cienc Tecnol Aliment 2003; 23:106-109. 